\title{
Medical Anniversaries of 1957
}

THE following list of anniversaries is presented in the hope that it may be helpful to those concerned with the arrangement of commemorative meetings, and with the organization of medico-historical exhibitions. No claim is made for completeness, the list representing no more than a selection from those anniversaries which can be discovered by the checking of dates of birth and death in some of the most easily accessible sources of information. It will be equally obvious that the anniversaries listed are not of equal importance. In some cases precise dates are not known, and in others a choice has had to be made between the conflicting dates given by 'standard authorities'.

\section{JANUARY}

ANDREW URE. Physician and chemist. 18 May 1778-2 Fanuary 1857.

Eugene Gley. French physiologist. 16 January 1857 -October 1930.

William Henry Horrogks. Bradford surgeon. 18 fanuary 1857-5 December $19 i 0$.

Vladimir Mihailovich Beghterev. Russian neurologist and psychiatrist. 20 January 1857-24 December 1927.

Pierre Felix Lagrange. French ophthalmologist. 22 January 1857-22 April rg28.

Mrs. Ethel Gordon Bedford-Fenwick. Founder of British Nurses' Association, etc. 26 January 1857-13 March 1947.

Francis Moore. Physician, astrologer and originator of Old Moore's Almanac. 29 Fanuary $1657-1715$ ?

\section{FEBRUARY}

Wilhelm Ludwig Johannsen. Danish botanist and geneticist. First to use the term 'gene'. 3 February 1857-II November 1927.

Henry Dwight Ghapin. American paediatrician. 4 February 1857-27 Fune 1942.

Sir Arthur Newsholme. Principal Medical Officer, Local Government Board. Io February 1857-17 May 1943.

Elisha Kent Kane. American naval surgeon and Arctic explorer. 3 February I820I6 February 1857 .

Jean Riolan. French physician and opponent of Harvey's views on the circulation. 20 February ${ }^{15} 80-19$ February 1657.

Heinrigh RUdolf Hertz. German physicist. 22 February I857-1 Fanuary I894.

Emile Gout. French psychologist and advocate of systematic auto-suggestion (Couéism). 26 February 1857-2 Fuly 1926.

Raphä̈l Anatole Emile Blanghard. French physician, parasitologist and medical historian. 28 February $1857-7$ February 1919.

\section{MARGH}

Alexis Boyer. French surgeon. I March I757-25 November I833.

JULIUS WAgner von Ja UREgG. Austrian neurologist and psychiatrist. 7 March 1857I October 1940. 


\section{Medical Anniversaries of 1957}

Fedor Krause. German surgeon. 1o March $1857-1937$ ?

ThEODORE TUFFier. French surgeon. 26 March 1857-27 October 1929.

Karl Pearson. Mathematician and biologist. 27 March 1857-27 April 1936.

\section{APRIL}

Henry Lewis Jones. British pioneer of electrotherapy. 5 April 1857-4 April rigr.

Franz Ziehl. German neurologist. 13 April 1857-7 April 1926.

Sir Vigtor Alexander Haden Horsley. London surgeon. 14 April 1857-16 July 1916.

Robert Bicke RSteth. Liverpool surgeon. I787-I7 April I857.

Ladislaus Bieganski. Polish physician and philosopher. 25 April 1857-29 January rgi7.

Giorgio Rattone. Italian pathologist. 25 April 1857-20 December 1929.

Eugen Ble ule R. Swiss psychiatrist. 30 April 1857-19 July 1939 .

Franģois Gueneau de Mussy. Paris physician. II June I774-30 April 1857.

\section{MAY}

Alessandro Lustig. Italian pathologist and bacteriologist. 5 May 1857-1937?

Louis BARD. French physician. Io May 1857-21 February 1930.

HeNdRiK ZWAardemake R. Netherlands physiologist. Io May 1857-19 September 1930.

MARShALl HALl. British physician and physiologist. 18 February I790-II May 1857.

Henry Herbert Donaldson. Director of Scientific Research, Wistar Institute. I2 May 1857-23 January 1938.

Sir Ron ALd Ross. Discoverer of the mosquito cycle in malaria. 13 May 1857-16 September 1932.

Robert Walter Doyne. Oxford ophthalmologist. 15 May 1857-30 August igi6.

Fortunato Liceto. Italian physician and author of the famous De Monstris, r6r3. 3 October 1577-17 May 1657 .

Wilhelm Gottlieb Tilesius von Tilenau. German physician and traveller. I7 July I769-17 May 1857 .

John Jaco B Abel. Johns Hopkins pharmacologist. 19 May 1857-26 May 1938.

Karl Gustav Lennande R. Swedish surgeon. 20 May 1857-15 March 1908.

William Gharles Wells. Physician and scientist. Author of the celebrated Essay on Dew, I814. 24 May I757-18 September 1817.

George Alder Blumer. American psychiatrist. 25 May 1857-25 April 1940.

WILliAM WOOD. London surgeon and zoologist. 1774-26 May 1857 .

John Howell. Army surgeon, later physician at Bristol. 21 October 1777-28 May 1857.

Antoine Germain Labarraque. French chemist; wrote on disinfection and on occupational diseases. 29 May $1757-9$ December 1850.

\section{JUNE}

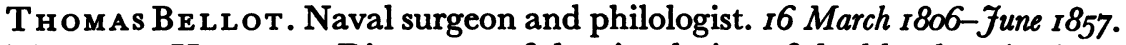

William HaR Vey. Discoverer of the circulation of the blood. I April 1578-3 fune 1657 .

Pierre Jean Georges Gabanis. French physician and philosopher. 5 June 1757-5 May 1808.

JAMIESON BOYd HuRRY. Reading physician; wrote on ancient Egyptian medicine and on vicious circles in disease. 8 June $1857-13$ February 1930.

Sir Robert Garswell. London physician and pathologist. 3 February 1793-15 June 1857 . 


\section{Medical Anniversaries of 1957}

\section{JULY}

Sir Frederic William Hewitt. London anaesthetist. 2 Fuly i857-6 January igi6.

Sir Alfred Henry Keogh. Director-General, Army Medical Services. 3 Fuly 185730 July 1936 .

John Templeton Bowen. Boston dermatologist. 8 July 1857-3 December 1940.

Alf Red Binet. French psychologist. II Fuly 1857-18 October I9II.

Gharles David Badham. Physician, priest and naturalist. 27 August 1805-14 7uly 1857.

Manuel Godorniuy Ferreras. Spanish physician. ?-I8 Fuly 1857.

James ER Nest LANe. London surgeon. 24 July $1857-4$ November 1926.

Friedrich S chrader. Professor of Medicine, Helmstedt. 30 fuly r657-r704.

John Alexander MacWilliam. Regius Professor of Physiology, Aberdeen. $3^{I}$ July 1857-13 January 1937 .

\section{AUGUST}

Otto Tiger Freer. Ghicago otolaryngologist. 8 August 1857-21 April 1932.

SAMUEL MEDLEy. Honorary member of the Medical Society of London and painter of the well-known picture of its founders. 22 March $1769-10$ August 1857.

Frederick John Smith. Physician and Lecturer on Forensic Medicine, London Hospital. I7 August 1857-30 April rgrg.

Emir. Bock. Austrian ophthalmologist. 19 August 1857-17 December 1916.

Thomas Robert Bradshaw. Liverpool physician. 29 August 1857-25 Fanuary 1927.

\section{SEPTEMBER}

Sir Gharles Mansfield Glarke. London obstetrician. 28 May 1782-7 September 1857•

Sir Gharles Pardey Luris. Director-General, Indian Medical Service. 9 September I857-22 October 1917 .

James Adey Ogle. Regius Professor of Medicine, Oxford. 22 October 1792-25 September 1857.

Gius e P P E My A. Italian paediatrician. 29 September 1857-5 February 1911.

\section{OGTOBER}

Grovanni MaRtinotti. Italian pathologist and medical historian. I October I857II October rg28.

Robert KeAte. Serjeant-Surgeon to William IV and Queen Victoria. 14 March I7772 October 1857 .

Jean Alban Bergonie. French radiologist and electrotherapist. 7 October 18572 January 1925.

O'Bryen Bellingham. Dublin surgeon. 12 December 1805-1I October 1857.

Samuel Tuke. Philanthropist and writer on the care of the insane. $3^{I}$ Fuly $1784-14$ October 1857.

Rene Antoine FerGhaut de Reaumur. French physicist and naturalist. 28 February I683-I7 October 1757 .

Roy al Whit man. American orthopaedic surgeon. 24 October 1857-19 August 1946.

Georges Gilles de la Tourette. French neurologist. 30 October 1857-26 May 1904.

Francesco Agrietti. Italian physician and medical historian. 3 I October 1757-3 May 1836.

Axel Munthe. Swedish physician; author of The Story of San Michele. 31 October 1857II February 1949. 


\section{Medical Anniversaries of 1957}

\section{NOVEMBER}

Alexander Kolisko. Austrian pathologist. 6 November 1857-23 February 1918.

Sir William Hale-White. London physician. 7 November 1857-26 February 1949.

Sir Arthur Glarke. Dublin physician. I773-9 November 1857 .

Samuel Herbert Habershon. London physician; friend of W. E. Gladstone. II November 1857-26 February 19I5.

Robert Willan. Physician and dermatologist. 12 November 1757-12 April 18r2.

Jose Ph BABINSKi. Paris neurologist. I7 November 1857-29 October 1932.

Sir Arthur Thomas Sloggett. Director-General, Army Medical Service. 24 November $1857-27$ November 1929 .

Sir Arghibald Edward Garrod. Regius Professor of Medicine, Oxford. 25 November I857-28 March 1936 .

Michael Bernhard Valentini. Professor of Medicine, Giessen. 26 November i657I8 March I729.

Sir Gharles Scott Sherrington. Oxford physiologist. 27 November 1857-4 March 1952.

TheOdor Escherich. German paediatrician and bacteriologist. 29 November I857I5 February IgII.

\section{DECEMBER}

Sir Robert Armstrong-Jones. London psychiatrist. 2 December 1857-30 Fanuary 1943.

Garl Koller. Austrian ophthalmologist; introduced cocaine as a local anaesthetic. 3 December 1857-21 March 1944 .

John Benjamin Murphy. Chicago surgeon. 21 December 1857-II August rgir6.

Vassili Vassilievich Stroganoff. Russian obstetrician; known for his writings on eclampsia. 29 December $1857-$ ?

Garl Benda. German pathologist and histologist. 30 December 1857-1933.

James Alexander Ad ams. Glasgow surgeon. 1857-28 December 1930.

John Atrins. Author of The Navy Surgeon (1732) and A Voyage to Guinea (1735). $1685-1757$.

Jean BAPtiste Lucien BA Udens. French military surgeon. 1804-1857.

ANGelo Gelli. Italian physician; historian of malaria. 1857-2 November 1914.

Hen Ri Gha Put. French surgeon. 1857-19r9.

NicGolo FALGUGCI. Italian physician. I357-I4II-I2.

Fran GeSGo GHil AR DUGGI. Italian radiologist. 1857-October 1924.

Johan N Hof F MANN. German physician. 1857-rgrg.

Simon P. Hullihen. American oral surgeon. Io December I810-1857.

John MAGintYre. Glasgow laryngologist; pioneer of X-ray cinematography. 185729 October 1928.

Mesuë the Elder (Johannes Damascenus). 777 or $780-857$.

Goncalo Fernandez de Oviedoy Valdes. Historiographer; wrote on syphilis and on materia medica of the New World. $147^{8-1557 .}$

Domenico Panaroli. Professor of Botany and Anatomy, Rome. ?-1657.

British Medical Journal assumed its present title, 3 January 1857.

Select Observations on English Bodies, by DR. JOHN HALL, Shakespeare's son-in-law, published by James Cooke, 1657 . 


\section{Medical Anniversaries of 1957}

Jонn HuXham's Dissertation on the Malignant Ulcerous Sore-Throat, 1757.

Medical Observations and Inquiries by a Society of Physicians in London, I757-84. 6 vols.

David Livingstone's Missionary Travels and Researches in South Africa, November 1857.

H. W. von Ziemssen's Die Electricität in der Medicin, 1857.

1257 Sorbonne founded.

1557 Gonville and Caius College, Cambridge, refounded.

I857 Danish Medical Association.

National Hospital for Diseases of the Heart.

New York Infirmary and College for Women (first hospital conducted wholly by women).

Royal Eye and Ear Hospital, Bradford.

University of Chicago.

Weston-super-Mare Hospital.

rgo7 King Edward's Hospital Fund for London established. 